\title{
Detection of Hydrophobic Contaminants at Water Surface by using Impedance Spectroscopy
}

\author{
Jorge H. Torres, Roshanak Gonzalez \\ Department of Bioengineering, \\ Florida Gulf Coast University, \\ 10501 FGCU Blvd South, Fort Myers, Florida 33965
}

\begin{abstract}
Organic compounds of various sources can contaminate water and be potentially toxic to humans and animals. We investigated the use of electrical impedance spectroscopy measurements as a method to detect small amounts of a group of organic compounds that are hydrophobic, stay at the water surface because of their low density, and can significantly alter the electrical conduction properties of the water they contaminate. We constructed a basic surface sensor for this specific purpose and used common mineral oil as a model hydrophobic organic contaminant. Our results showed significant changes in AC electrical properties of the liquid surface in the presence of the hydrophobic model contaminant. The complex impedance obtained from the impedance spectroscopy measurements are presented as plots of magnitude and phase as well as a plot of real vs. imaginary components for various AC frequencies. The data showed significant changes in resistive and capacitive components of the impedance for contaminated water compared with pure water. The ultimate goal of this research is to determine the ability of the method to detect small contaminant concentrations.
\end{abstract}

Key words: Hhydrophobic compounds, surface sensing, water contaminant, complex electrical impedance, impedance spectroscopy

\section{INTRODUCTION}

Many water contaminants are organic compounds potentially toxic for humans and animals. Some of those organic contaminants are low density hydrophobic compounds that stay at the surface of water ponds and other water sources. In small concentrations, those contaminants may be difficult to detect as they may form a thin continuous or broken layer at the water surface.

AC impedance spectroscopy has been used to measure electrical conductivity in various media including liquid solutions [1-3]. Normally, these measurements are performed with electrodes embedded in the liquid itself. In the case of water, impedance spectroscopy has been used to detect different types of contaminants including organic compounds but, usually, the organic contaminants are hydrophilic in nature or have polar components that allow them to mix with the water to form a solution. For this type of solutions, and utilizing electrodes embedded in the water, impedance spectroscopy is one tool that has been used to detect the presence of these contaminants [4,5]. The complication arises when the organic contaminant is hydrophobic and does not mix with the water. In these circumstances, regular electrodes and impedance spectroscopy measurements are inadequate to detect the contaminant as it clumps in small micelles surrounded by the water molecules. Detection becomes even harder when the organic compound has density lower than water, a very common situation, and moves to the liquid surface. In small concentrations, the contaminant forms a very thin layer that brakes in patches all over the water surface. Construction of an appropriate sensor and electrical conductivity measurements become a challenge. Although optical techniques could be used to detect the presence of organic films at water surfaces, they tend to require some elaborate or expensive instrumentation [6,7]. Impedance spectroscopy may provide a simple inexpensive alternative that may allow detection of a broad range of hydrophobic molecules.

In the impedance spectroscopy technique, a series of $\mathrm{AC}$ voltage pulses at different frequencies are applied and the corresponding resulting current is measured for each frequency. From the voltage and current amplitudes and the phase shift in the current with respect to voltage, the complex impedance can be calculated. This complex impedance contains two components: the real part corresponding to the electrical resistance and the imaginary part corresponding to the electrical reactance due to a capacitive effect.

For the study presented in this paper, a simple custommade sensor for surface detection was constructed and the impedance spectroscopy technique was used to determine if it could be used to detect hydrophobic organic contaminants at the water surface. Here, we looked for variations in the complex electrical impedance along the contaminant built-in surface layer when compared to the complex impedance at the surface of clean water. And in this case, the electrical conduction along the layer is investigated instead of the most common conduction across the layer that is measured in other applications of the technique.

\section{MATERIALS AND METHODS}

\subsection{Surface Sensor}

A simple custom-made sensor was constructed with two titanium electrode disks, of approximately $0.7-1.0 \mathrm{~cm}$ in diameter, attached to the bottom of a small Styrofoam block as can be seen in Figure 1. The disks were separated by approximately $1 \mathrm{~cm}$ distance. Titanium wires were 
welded to the lateral edges of the electrode disks so the wires came out at the top of the Styrofoam block and contacted two titanium rods that served as final contacts for the connections to the measurement instrument as can be seen in Figure 2. The bottom of the Styrofoam block with the electrodes was placed just floating on the surface of the liquid whose impedance was to be measured. The liquid, composed of either clean water or water plus organic contaminant, was contained in a standard petri dish as seen in the figure.

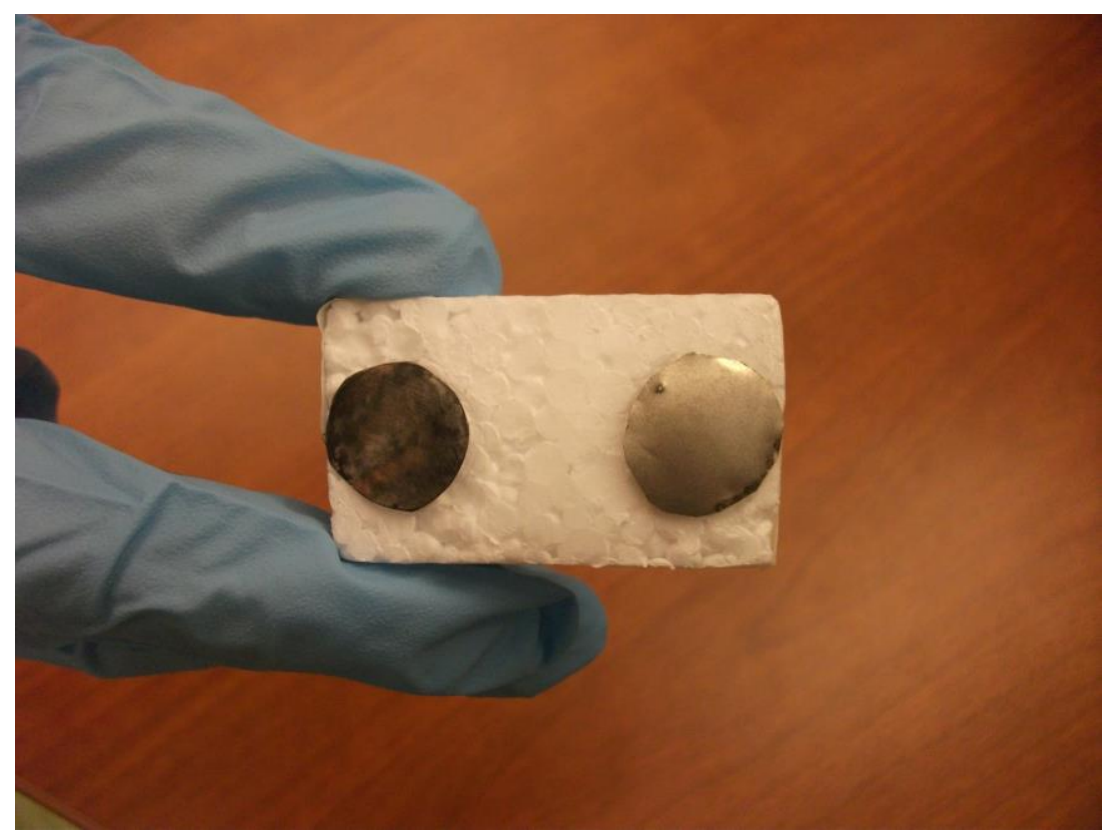

Figure 1. Picture of the basic surface sensor constructed. Titanium electrodes attached to a Styrofoam block.

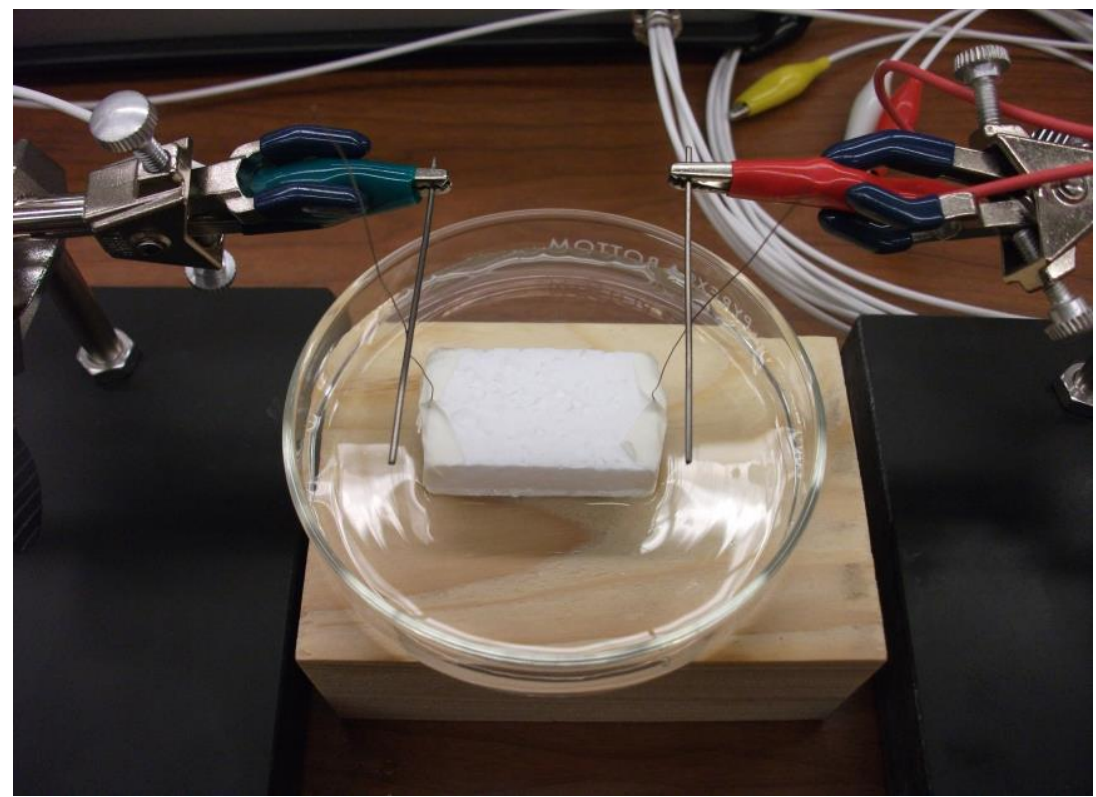

Figure 2. Picture of the experimental setup showing the placement of the Styrofoam block with electrodes in contact with the liquid to be tested.

\subsection{Hydrophobic Organic Contaminant and Emulsion}

$60 \mathrm{ml}$ of deionized water were placed inside the petri dish as control. Deionized water was used to avoid the effects of ions on the electrical conductivity of the water. For the hydrophobic organic contaminant-water mixture (actually emulsion), a volume of 1 or $2 \mathrm{ml}$ of pharmaceutical grade mineral oil was added and rigorously stirred until a uniform film was formed. When only $1 \mathrm{ml}$ was added, a consistent, stable, lasting uniform film was hard to achieve, while the addition of the $2 \mathrm{ml}$ volume consistently provided a desired lasting uniform film. For this latter case, the thickness of the oil layer was calculated to be $314 \mu \mathrm{m}$, based on the petri dish dimensions. The pharmaceutical grade mineral oil was considered a safe representative of a group of hydrophobic organic compounds that could be used as model contaminants for the impedance tests. This mineral oil is a mixture of higher alkanes derived from petroleum. 
It is safe, inert, easy to use, highly hydrophobic, and has a density around $0.85 \mathrm{~g} / \mathrm{ml}$.

\subsection{Measurement Instrumentation and Procedure}

An Electrochemical Workstation model chi660C made by $\mathrm{CH}$ Instruments was utilized to measure the $\mathrm{AC}$ impedance between the two titanium electrodes. AC pulses of $1 \mathrm{~V}$ were applied at frequencies between 1 and 10,000 $\mathrm{Hz}$. Repetitive runs in groups of 5 sweeping through the same range of frequencies were applied. Plots of impedance magnitude and impedance phase were obtained, together with a Nyquist complex plot of real vs. imaginary impedance components. Test runs were done for the deionized water control and for the water with the addition of the mineral oil.

\section{RESULTS}

\subsection{Experimental Results}

For the added $1 \mathrm{ml}$ volume of mineral oil, the results were not consistent as the uniform surface layer was not stable and broke into patches. For the size of electrodes used and the distance between them, it was unclear if the electrodes were partially touching the oil and partially the water. In this case, smaller electrodes with shorter separation could work better. We stuck to our sensor dimensions, however, as the added $2 \mathrm{ml}$ volume of mineral oil produced a very stable surface film and the measurements turned out to be extremely consistent and reproducible. The graphs below correspond to the comparative results of the clean water control with the contaminated water having the $2 \mathrm{ml}$ of mineral oil added to it. When measurements were done within 2 hours or less between each other, the results were so consistent and reproducible that basically no deviation of the curves was observed in the plots run after run of the frequency sweep. However, when the Styrofoam was left on the oil layer more than 2 hours, it progressively absorbed oil, sank into the oil layer and could not be used any more. That was a floating material issue that can be solved in the future with a different material for longer use. Used within two hours, the Styrofoam worked well as a light floating substrate holding the electrodes at the surface of the liquid under test.

Figures 3, 4, and 5 below show comparative impedance results for the clean water control and the water containing a surface layer of mineral oil. In all these graphs, $2 \mathrm{ml}$ of mineral oil was added to $60 \mathrm{ml}$ of water. Figures 3 and 4 present the magnitude and the phase, respectively, of the complex impedance as a function of frequency. Magnitude and frequency are plotted in log scales. Both magnitude and phase stabilize at a frequency around $160 \mathrm{~Hz}(\log =$ 2.2). In Figure 4, the dip seen in the plot of the phase between 250 and $1,000 \mathrm{~Hz}(\log =2.4$ to 3$)$ is, unfortunately, an artifact generated by the instrument and needs to be discounted, as the actual phase sets around zero degrees. In this phase graph, all the phase angles (see Y axis label) are negative because of the capacitive effect on the impedance. In Figure 3, the impedance magnitude is consistently higher for mineral oil when compared to the water control for all frequencies. In Figure 4, the negative phase value of the impedance for mineral oil is higher than that for the water control for frequencies until $160 \mathrm{~Hz}(\log$ $=2.2$ ) when both phases go to zero. According to these graphs, both impedance magnitude and phase change with the presence of the mineral oil surface layer.

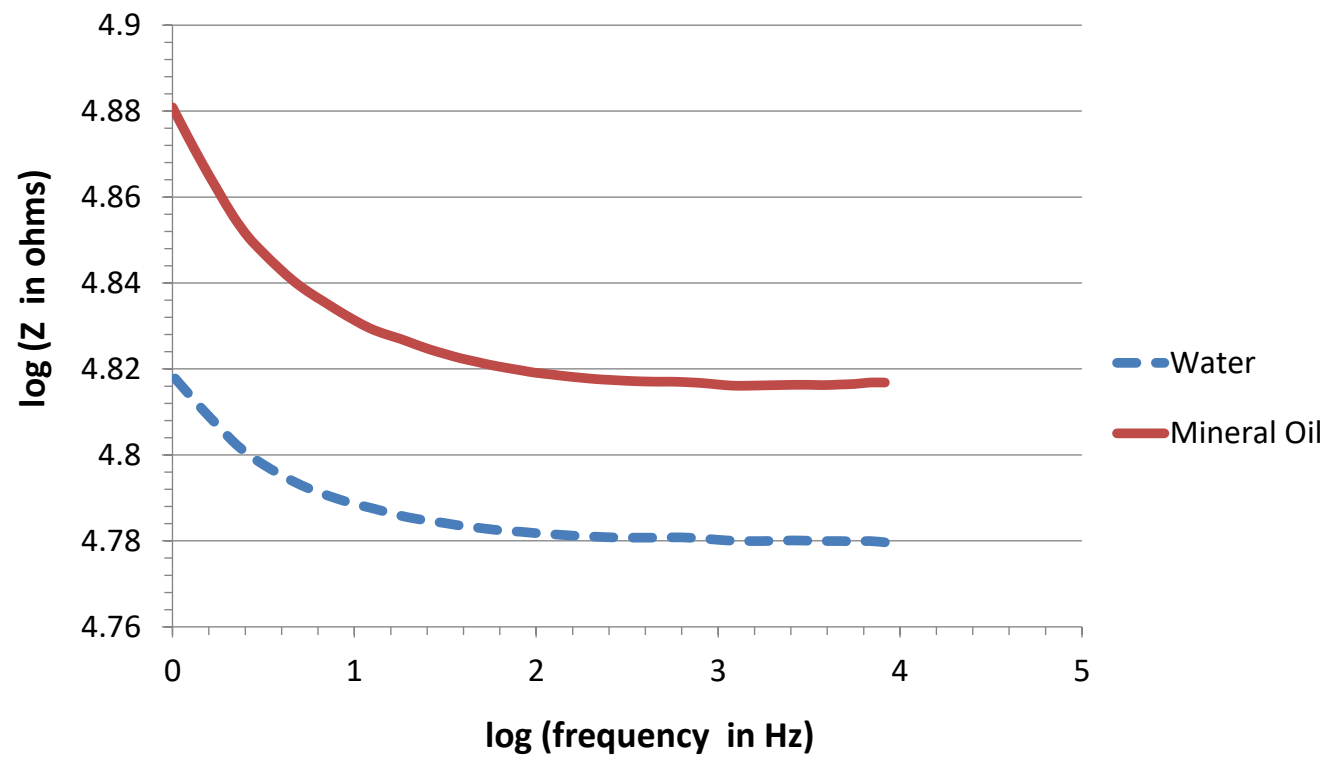

Figure 3. Impedance magnitude as a function of frequency. The impedance increases with the addition of the mineral oil for all frequencies. 


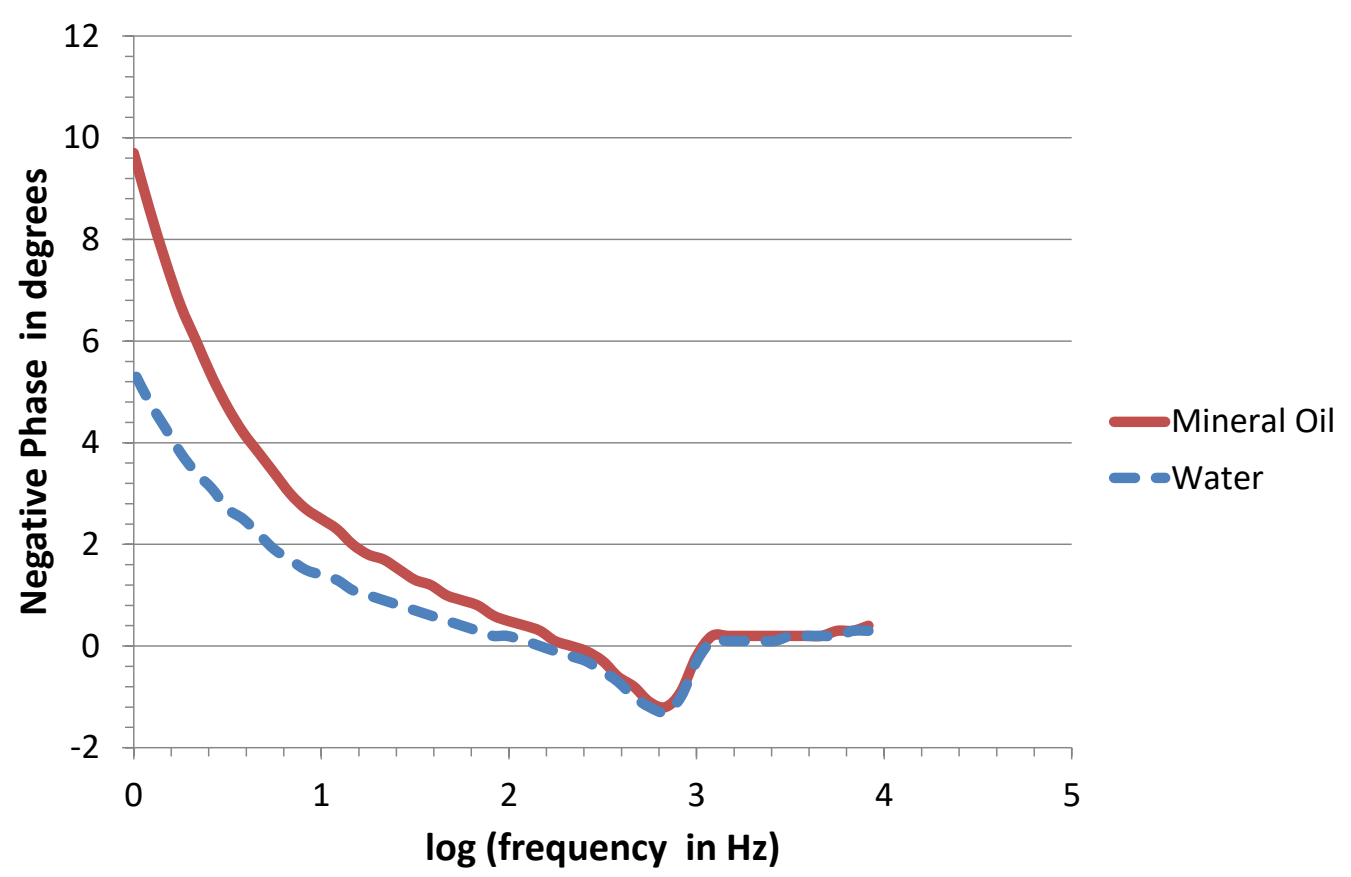

Figure 4. Impedance phase as a function of frequency. The phase increases with the addition of the mineral oil for the lower frequencies. The dip below the zero line is an instrumentation artifact. The actual phase stops at zero.

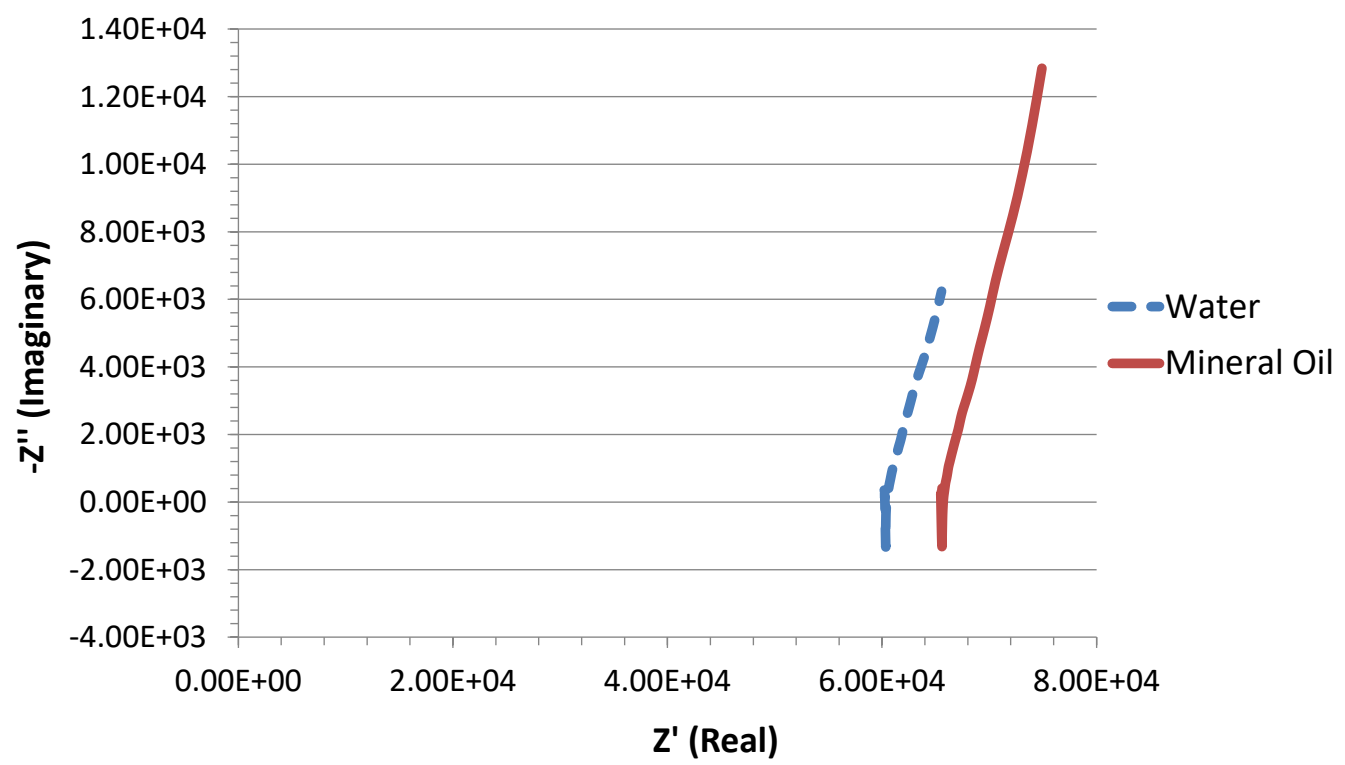

Figure 5. Nyquist complex plot depicting the real vs. the imaginary components of the impedance. The addition of the mineral oil increases both the real and the imaginary components. The short negative lines are due to instrument artifact as the imaginary impedance stops at zero.

In Figure 5, the Nyquist complex plot of real component vs. imaginary component of the impedance is presented. This Nyquist plot is obtained from the magnitude and phase presented in the previous figures. The real component corresponds to the purely resistive effect and the imaginary component corresponds to the capacitive effect occurring in the layer. The presence of the mineral oil shifts both the real and the imaginary components of the impedance towards higher values. The dip observed below zero for the imaginary component of the impedance is again due to the instrument-generated artifact in the phase that was mentioned before. In reality, the imaginary component stops at zero at high frequencies as the capacitance produces a virtual short with the reactive 
impedance becoming zero. Figure 5 also shows that for both, water and mineral oil, there is a variation in the resistive (real) component with frequency. Each curve looks like a straight line tilted from a vertical line. values at four selected frequencies, increasing by order of magnitude, obtained for the case of water only and for the case of the mineral oil layer covering the surface. All values are higher for the mineral oil layer.

Table 1 below presents real component (Z'), imaginary component (Z”), and total magnitude (|Z|) impedance

\begin{tabular}{|l|l|l|l|l|l|l|}
\hline & \multicolumn{3}{|l|}{ Water Only Control } & \multicolumn{3}{l|}{ Mineral Oil Layer at Surface } \\
\hline Freq(Hz) & $\mathrm{Z}^{\prime}(\mathrm{k} \Omega)$ & $\mathrm{Z}^{\prime}(\mathrm{k} \Omega)$ & $|\mathrm{Z}| \mathrm{k} \Omega)$ & $\mathrm{Z}^{\prime}(\mathrm{k} \Omega)$ & $\mathrm{Z}^{\prime \prime}(\mathrm{k} \Omega)$ & $|\mathrm{Z}| \mathrm{k} \Omega)$ \\
\hline 1 & 65.6 & 6.24 & 65.8 & 74.9 & 12.8 & 76.0 \\
\hline 10 & 61.5 & 1.51 & 61.5 & 67.8 & 2.94 & 67.8 \\
\hline 100 & 60.5 & 0.184 & 60.5 & 65.9 & 0.583 & 65.9 \\
\hline 1000 & 60.3 & 0.136 & 60.3 & 65.5 & 0.231 & 65.5 \\
\hline
\end{tabular}

Table 1. Real component, imaginary component, and magnitude impedance values at four selected frequencies, increasing by order of magnitude, for water only and for the mineral oil layer at the surface.

\subsection{Electrical Model Based on Experimental Data}

The fact that, in the graph of Figure 5, both lines are not vertical but tilted gives support to the traditional electrical model used for the impedance spectroscopy with a parallel resistive component (parallel with a capacitor) and a series resistive component. This model is depicted in Figure 6. This model is used for impedance measurements across a layer, with electrodes at both sides of the layer, but it appears appropriate to be used for these impedance measurements along the layer itself.

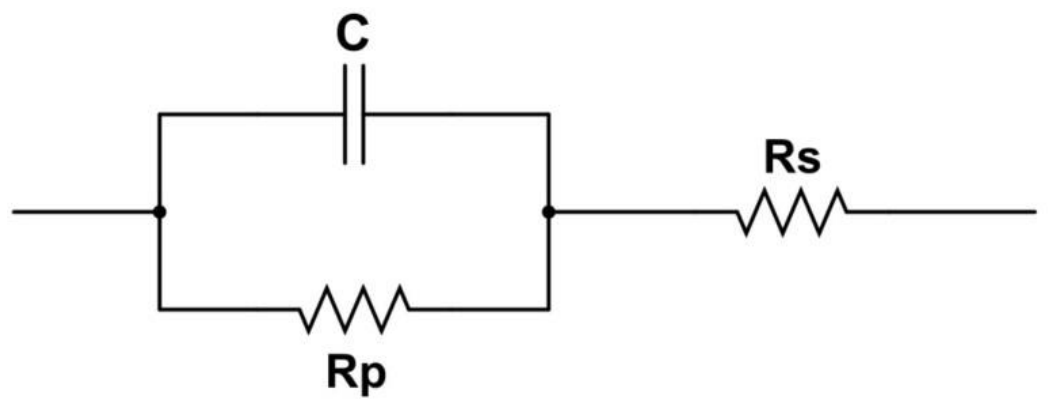

Figure 6. Electrical model corresponding to the impedance spectroscopy results. C is capacitance, Rp is the parallel resistance, and Rs is the series resistance.

Knowing that the capacitor becomes an open circuit as frequency approaches zero (or DC) and a short circuit at very high frequencies, the parallel resistance $(\mathrm{Rp})$ and the series resistance (Rs) could be determined from experimental data. Although the instrumentation in this work did not include DC, and only frequencies from $1 \mathrm{~Hz}$ to $8,250 \mathrm{~Hz}$ were actually part of the sweep, approximate values for $\mathrm{Rp}$ and $\mathrm{Rs}$ were obtained using $1 \mathrm{~Hz}$ as the lowest frequency and $1,000 \mathrm{~Hz}$ as the highest frequency. This latter high end frequency worked fine since the real part of the impedance did not have any significant change after 1,000 Hz. The approximate values of the resistances were obtained as:

Rs $=$ real impedance at $1,000 \mathrm{~Hz}$

$\mathrm{Rp}=$ real impedance at $1 \mathrm{~Hz}-$ real impedance at $1,000 \mathrm{~Hz}$

Table 2 below compares the approximated values of Rs and $\mathrm{Rp}$ of water only with those of the mineral oil layer. The values are higher for the mineral oil layer as expected.

\begin{tabular}{|l|l|l|l|}
\hline \multicolumn{2}{|l|}{ Water Only Control } & \multicolumn{2}{l|}{ Mineral Oil Layer } \\
\hline $\mathrm{Rs}(\mathrm{k} \Omega)$ & $\mathrm{Rp}(\mathrm{k} \Omega)$ & $\mathrm{Rs}(\mathrm{k} \Omega)$ & $\mathrm{Rp}(\mathrm{k} \Omega)$ \\
\hline 60.3 & 5.3 & 65.5 & 9.4 \\
\hline
\end{tabular}

Table 2. Approximated values of the parallel ( $\mathrm{Rp})$ and series (Rs) resistances for water only and mineral oil layer.

The approximate capacitance can be calculated from the above resistance values and the total complex impedance provided by the instrumentation. Based on the electrical model of Figure 6, the total complex impedance $(Z)$ will be equal to:

$$
Z=\frac{(R p)(1 / j \omega C)}{R p+(1 / j \omega C)}+R s
$$

Where $\omega=2 \pi f$, being $f$ the frequency in Hertz.

Working the above equation to express the complex impedance in the conventional rectangular form of real plus imaginary components, we obtain: 


$$
Z=\left[R s+\frac{R p}{1+\omega^{2} C^{2} R p^{2}}\right]-j\left[\frac{\omega C R p^{2}}{1+\omega^{2} C^{2} R p^{2}}\right]
$$

Where the first term in brackets corresponds to the real component (Z') and the second term in brackets corresponds to the imaginary component (Z'). Using the real component, we can solve for capacitance (C) as:

$$
C=\frac{\sqrt{\frac{R p}{\operatorname{Real}\{Z\}-R s}-1}}{2 \pi f R p}
$$

From this last expression and the experimental data, capacitance can be calculated. At low frequencies, the capacitance has more effect on the impedance (see Figure 3 ) and the results are expected to be closer to the actual capacitance value. Table 3 presents the impedance measurements for water only and for the mineral oil layer at two low frequencies: 3.162 and $5.62 \mathrm{~Hz}(\log$ of frequency 0.5 and 0.75 , respectively, in Figure 3). Table 4 presents the corresponding calculated values of the capacitance at those two frequencies. The calculated capacitance of the mineral oil layer is almost half of that of the water control. And as a result, the imaginary part of the impedance is almost twice for the mineral oil layer compared to the water control.

\begin{tabular}{|l|l|l|l|l|l|l|}
\hline & \multicolumn{3}{|l|}{ Water Only Control } & \multicolumn{3}{l|}{ Mineral Oil Layer at Surface } \\
\hline Freq $(\mathrm{Hz})$ & $\mathrm{Z}^{\prime}(\mathrm{k} \Omega)$ & $\mathrm{Z}^{\prime}(\mathrm{k} \Omega)$ & $|\mathrm{Z}|(\mathrm{k} \Omega)$ & $\mathrm{Z}^{\prime}(\mathrm{k} \Omega)$ & $\mathrm{Z}^{\prime}(\mathrm{k} \Omega)$ & $|\mathrm{Z}|(\mathrm{k} \Omega)$ \\
\hline 3.162 & 62.7 & 3.01 & 62.8 & 70.1 & 5.8 & 70.3 \\
\hline 5.62 & 61.9 & 2.1 & 62.0 & 68.7 & 4.07 & 68.8 \\
\hline
\end{tabular}

Table 3. Impedances measured for two low frequencies used to calculate capacitance.

\begin{tabular}{|l|l|l|l|}
\hline & Water & Oil Layer & Ratio of capacitances \\
\hline Freq $(\mathrm{Hz})$ & $\mathrm{C}(\mu \mathrm{F})$ & $\mathrm{C}(\mu \mathrm{F})$ & $\mathrm{C}$ water $/ \mathrm{C}$ oil \\
\hline 3.162 & 10.44 & 5.47 & 1.91 \\
\hline 5.62 & 8.125 & 4.194 & 1.94 \\
\hline
\end{tabular}

Table 4. Calculated capacitance for the two frequency values of Table 3.

\section{DISCUSSION}

Hydrophobic organic compounds contaminating water sources could be potentially toxic to humans and animals. When contamination is suspected, samples could be taken for posterior analysis by chromatography or other methods. However, in situ detection could make the process simpler and faster. But the in situ detection of small quantities of hydrophobic organic compounds as contaminants resting on the surface of standing water sources is challenging. Optical techniques could be used but the instrumentation required could be elaborate and expensive. Impedance spectroscopy measurements may provide an alternative, but the technique requires an appropriate sensor and feasibility tests need to be performed for surface applications. In this study, a simple sensor for electrical impedance measurements of surface films was constructed, a hydrophobic organic compound was used as contaminant model producing a surface film, and impedance spectroscopy measurements were performed to test the feasibility of the method for the aforementioned sensing application.

The results of this study show that the impedance spectroscopy technique could be used for detection of a surface film made of a hydrophobic organic compound resting as surface contaminant of standing water sources. Here, the addition of mineral oil, used as contaminant model, to water produced significant changes in the complex electrical impedance. The mineral oil increased both the resistive and capacitive components of the impedance. These changes were expected for a hydrophobic less conductive compound compared to water, but needed to be seen when applied to films on water surface. The hydrophobic contaminant increases the resistance and lowers the capacitance. The latter reduction in capacitance increases the reactance or imaginary component of the impedance.

In this investigation, $2 \mathrm{ml}$ of mineral oil were added to 60 $\mathrm{ml}$ of water in a petri dish producing a stable uniform film of the mineral oil at the surface of the water. However, smaller volumes of the mineral oil ( $1 \mathrm{ml}$ or less) were inadequate for our detection most likely due to the layer formed at the surface being too thin or non-uniform for the sensor we constructed. This sensor was simple, easy to make, and very cheap. Improvement in sensor construction and reduction in the sensor dimensions will be the key to improved detection capabilities for smaller quantities and lower concentrations of the hydrophobic contaminant, and that should be the goal for future work on this line of detection.

\section{CONCLUSION}

The impedance spectroscopy technique with an appropriate sensor construction and placing could be used to detect the presence of a hydrophobic organic film as contaminant on the surface of standing water sources. A cheap sensor of simple construction can be made to be used with this detection technique. 


\section{ACKNOWLEDGEMENTS}

We want to thank Ms. Lauren Estridge-Zwanzig for her assistance with some of the measurements.

\section{REFERENCES}

[1] Byoung-Yong Chang and Su-Moon Park, Electrochemical impedance spectroscopy, Annual Review of Analytical Chemistry, Vol 3, pp.207-229, 2010.

[2] Digby D. Macdonald, Reflections on the history of electrochemical impedance spectroscopy, Electrochimica Acta, 51(8-9), pp.1376-1388, Jan 2006.

[3] J. Ross Macdonald and William B. Johnson, Fundamentals of Impedance Spectroscopy, in "Impedance Spectroscopy: Theory, Experiment, and Applications", Evgenij Barsoukov and J. Ross Macdonald, Eds. April 2018.

[4] F. Lisdat and D. Schafer, The use of electrochemical impedance spectroscopy for biosensing, Analytical and Bioanalytical Chemistry, July 2008, 391:1555.

[5] Asif I Zia et al., Technique for rapid detection of phthalates in water and beverages, Journal of Food Engineering, 116(2), pp. 515-523, May 2013.

[6] A.A. Ionin, D.V. Mokrousova, L.V. Seleznev, D.V. Sinitsyn, E.S. Sunchugasheva, Detection of thin oil films on the water surface with the help of UV filaments, Atmospheric and Oceanic Optics, 29(4), pp. 339-341, July 2016.

[7] S. Patsayeva, V. Yuzhakov, V. Varlamov, R. Barbini, R. Fantoni, C. Frasanito, and A. Palucci, Laser spectroscopy of mineral oils on the water surface, Proceedings of EARSeLSIG-Workshop LIDAR, Dresden/FRG, June 16-17, 2000. 